Michael Alex

\title{
Rückfallhäufigkeit und langer Beobachtungszeitraum - Die Wahrscheinlichkeit des Todes potentieller Rückfalltäter ist ebenso hoch wie das Risiko eines erneuten Gewalt- oder Sexualdelikts
}

\section{A. Die Bochumer Untersuchung zur nachträglichen Sicherungsverwahrung}

In den Jahren 2007 bis 2009 wurde an der Ruhr-Universität Bochum, Lehrstuhl für Kriminologie, ein Forschungsvorhaben zur nachträglichen Sicherungsverwahrung durchgeführt. Das Projekt befasste sich mit der Legalbewährung von Haftentlassenen, bei denen Anträgen auf Anordnung nachträglicher Sicherungsverwahrung gemäß $\$ 66$ b StGB (ggf. vor dessen Inkrafttreten nach Straftäter-Unterbringungsgesetzen der Bundesländer Baden-Württemberg, Bayern, Sachsen-Anhalt, Thüringen, Niedersachsen) letztlich infolge der restriktiven Auslegung der neuen Vorschriften durch die Gerichte nicht stattgegeben worden ist und die deshalb in der Zeit von Januar 2002 bis zum 31.12.2006 aus der Haft entlassen worden waren. Obwohl Justizvollzugsanstalten und/oder Staatsanwaltschaft bei den betreffenden Strafgefangenen vor Ende des Vollzugs der Freiheitstrafe eine erhebliche Gefährlichkeit für die Allgemeinheit angenommen hatten und diese Annahme in vielen Fällen von Sachverständigen gestützt wurde, lehnten die Gerichte nach Vorgaben von Bundesgerichtshof und Bundesverfassungsgericht (BGH St 50, 121; BVerfG NStZ 2007, 87 ff.) in vielen Fällen die nachträgliche Anordnung von Sicherungsverwahrung mit der Begründung ab, die entsprechenden Tatsachen seien nicht erst während der Haft erkennbar gewesen bzw. seien nicht erheblich für die Beurteilung der Gefährlichkeit für die Allgemeinheit. Infolgedessen sind bis zum 31.12.2006 bundesweit etwa 115 vermeintlich hoch gefährliche Gefangene aus dem Strafvollzug entlassen worden. Die Bochumer Untersuchung hatte zum Ziel, anhand aktueller Auszüge aus dem Bundeszentralregister die Legalbewährung dieser Gruppe zu überprüfen. Mit Unterstützung des Justizministeriums des Landes Nordrhein-Westfalen und der Justizverwaltungen der übrigen Bundesländer (Ausnahme Bremen) war es möglich, die für den Abgleich erforderlichen Daten von 77 Haftentlassenen zu erlangen und darüber hinaus Einsicht in die Vollstreckungsunterlagen zu erhalten. Die Bundeszentralregisterauszüge wurden vom Bundesamt für Justiz ab Ende Juni 2008 übersandt und ergaben zunächst folgendes Bild: 
Strafhöbe bei erneuter Verurteilung $(n=27)$

\begin{tabular}{|l|l|l|l|l|l|l|}
\hline \multicolumn{2}{|l|}{ Erneute Verurteilung zu } & $\begin{array}{l}\text { Keine erneute Eintragung } \\
\text { bis 30.06.2008 }\end{array}$ \\
\hline Geldstrafe & $\begin{array}{l}\text { Freiheitsstrafe } \\
\text { m. Bewährung }\end{array}$ & \multicolumn{3}{|l|}{ Freiheitsstrafe o. Bewährung } \\
\cline { 3 - 6 } & & $<1 \mathrm{~J}$ & $\leq 2 \mathrm{~J}$ & $>2 \mathrm{~J}$ & SV & \\
\hline 10 & 5 & 3 & 2 & 4 & 3 & 50 \\
\hline
\end{tabular}

Von den Freiheitsstrafen, die nicht zur Bewährung ausgesetzt wurden, betrafen fünf eher Bagatelldelikte (dreimal Diebstahl, einmal Körperverletzung, einmal Betrug), die mit Freiheitsstrafen von vier Monaten bis zu einem Jahr und vier Monaten geahndet wurden. Einzelheiten zu den sieben schwerwiegenderen Rückfalldelikten (Freiheitsstrafe von mehr als 2 Jahren und damit nicht mehr zur Bewährung aussetzbar) sind in der folgenden Übersicht dargestellt:

\section{Index- und Rückfalldelikt bei Verurteilung zu Freibeitsstrafe von mebr als zwei Jabren und Strafmaß}

\begin{tabular}{|c|c|c|}
\hline Indexdelikt & Rückfalldelikt & Strafmaß \\
\hline Vergewaltigung & $\begin{array}{c}\text { Gemeinschaftlicher Raub, gef. } \\
\text { Körperverletzung }\end{array}$ & 2 Jahre und 2 Monate \\
\hline $\begin{array}{c}\text { Versuchte Vergewalti- } \\
\text { gung }\end{array}$ & Gemeinschaftl. Diebstahl & 2 Jahre und 6 Monate \\
\hline $\begin{array}{c}\text { Sexuelle Nötigung } \\
\text { Diebstahl, Fahren ohne } \\
\text { Fahrerlaubn. }\end{array}$ & $\begin{array}{c}\text { Sexuelle Nötigung, Körperverlet- } \\
\text { zung }\end{array}$ & 3 Jahre und Sicherungsverwahrung \\
\hline $\begin{array}{c}\text { Diebstahl, Widerstand, } \\
\text { WaffG }\end{array}$ & $\begin{array}{c}\text { Betrug, Einbruchsdiebst., Urkun- } \\
\text { denfälschung }\end{array}$ & 3 Jahre und 6 Monate \\
\hline $\begin{array}{c}\text { Schw. sex. Missbr. Von } \\
\text { Kindern }\end{array}$ & $\begin{array}{c}\text { Schw. sex. Missbrauch von Kin- } \\
\text { dern }\end{array}$ & 4 Jahre \\
\hline $\begin{array}{c}\text { Schwere räub. Erpres- } \\
\text { sung }\end{array}$ & $\begin{array}{c}\text { Schwere räub. Erpressung } \\
\text { BtMG }\end{array}$ & Sicherungsverwahrung \\
\hline
\end{tabular}

Vier Delikte sind Katalogtaten des $\$ 66 \mathrm{~b}$ StGB, erreichen also die dort geforderte Erheblichkeit für die Integrität der Opfer, wobei es sich in drei Fällen um einschlägige Rückfalldelinquenz handelte. Die Gesamtergebnisse der Untersuchung sind mittlerweile 
in unterschiedlicher Form veröffentlicht ${ }^{1}$. Einschränkend war bereits seinerzeit darauf hingewiesen worden, dass zum Erhebungszeitpunkt erst etwa 30\% der Probanden $(n=21)$ vor mehr als drei Jahren aus der Haft entlassen worden waren und sechs der zwölf Verurteilungen zu unbedingten Freiheitsstrafen auf diese kleine Gruppe entfielen. Durchschnittlich hatten sich die Probanden zum Erhebungszeitpunkt 33 Monate in Freiheit befunden, 34 (44\%) allerdings weniger als $2 \frac{1}{2} 2$ Jahre. Der relativ kurze Katamnesezeitraum bei einem Teil der Probanden war denn auch ein wesentlicher Kritikpunkt an den Ergebnissen der Rückfalluntersuchung. So gab die bayerische Justizministerin nach der ersten Veröffentlichung vorläufiger Ergebnisse am 11.09.2008 eine Pressemitteilung (Nr. 149/08) heraus, in der es u.a. heißt: „Außerdem kann die nachträgliche Sicherungsverwahrung überhaupt erst seit Juli 2004 verhängt werden. Die Studie konnte also nur Aussagen zur Rückfälligkeit von Tätern treffen, die seit höchstens vier Jahren auf freiem Fuß sind. Das ist für eine Beurteilung der nachträglichen Sicherungsverwahrung einfach zu kurz! Auch wer im fünften Jahr wieder ein Gewaltdelikt begeht, hat sich als gefährlich erwiesen!“

Diese Aussage ist zwar insoweit unzutreffend, als sich unter den Probanden auch sieben befanden, die nach den Straftäter-Unterbringungsgesetzen einzelner Bundesländer weiterhin inhaftiert bleiben sollten und vor Inkrafttreten von $\$ 66 \mathrm{~b} \mathrm{StGB}$ zwischen 2002 und dem 30.06.2004 entlassen worden waren, greift aber die Problematik kurzer Zeiträume zwischen Haftentlassung und Erhebung von Rückfalldaten auf, die bereits in dem Antrag vom 09.04.2008 an das Bundesamt für Justiz auf Auskunftserteilung berücksichtigt worden war. Darin war bereits angekündigt, dass unter Berücksichtigung der Ergebnisse der Rückfallforschung ${ }^{2}$ die Legalbewährung fünf Jahre nach der Haftentlassung noch einmal anhand eines weiteren Auszugs aus dem Bundeszentralregister kontrolliert werden sollte.

\section{B. Die Wiederholung des Abgleichs mit dem Bundeszentralregister im Jabre 2013}

Die Erteilung der im Juli 2011 beantragten wiederholten Auskünfte aus dem Bundeszentralregister verzögerte sich infolge der seit dem 01.01.2012 geltenden Neufassung des $\$ 42$ a BZRG und der darin verankerten erweiterten Datenschutzregelung um zwei Jahre und erfolgte erst Ende Juni 2013. Zu diesem Zeitpunkt waren acht der ursprünglich 77 Probanden bereits verstorben, wobei mindestens drei von ihnen zuvor noch zu geringfügigeren Freiheitsstrafen (in zwei Fällen zur Bewährung ausgesetzt) verurteilt worden

1 Alex NK 4/2008, 150-153; Feltes/Alex 2010, Kriminalpolitische und kriminologische Probleme der Sicherungsverwahrung, in: Dölling u.a.(Hrsg), Festschrift für Schöch, 733-754; Alex 2010, Nachträgliche Sicherungsverwahrung - ein rechtsstaatliches und kriminalpolitisches Debakel; Alex/Feltes Forum Strafvollzug 3/2010, 159-163; Alex Forensische Psychiatrie, Psychologie, Kriminologie 4/2011, 244-252; Alex 2012, Rechtsstaatliche Kapitulation. Das Beispiel der nachträglichen Sicherungsverwahrung, in: Strafverteidigervereinigungen, Organisationsbüro (Hrsg.), Abschied von der Wahrheitssuche, 125-146.

2 Jeble et al. 2003. 
waren. Für die verbliebenen 69 Probanden ergab sich in einem Zeitraum von 6 1/2 bis 13 Jahren nach der Haftentlassung folgendes Gesamtbild:

$$
\text { Strafhöhe bei erneuter Verurteilung }(n=42)
$$

\begin{tabular}{|c|c|c|c|c|c|l|}
\hline \multicolumn{2}{|l|}{ Erneute Verurteilung zu } & \multicolumn{2}{l|}{$\begin{array}{l}\text { Keine erneute Eintragung } \\
\text { bis 30.06.2013 }\end{array}$} \\
\hline Geldstrafe & $\begin{array}{l}\text { Freiheitsstrafe } \\
\text { m. Bewährung }\end{array}$ & \multicolumn{3}{|l|}{ Freiheitsstrafe o. Bewährung } & \\
\cline { 3 - 6 } & & $<1 \mathrm{~J}$ & $\leq 2 \mathrm{~J}$ & $>2 \mathrm{~J}$ & SV & \\
\hline 12 & 7 & 6 & 3 & 5 & 9 & 27 \\
\hline
\end{tabular}

Eine Steigerung der Rückfalldelinquenz lässt sich vor allem im Bereich der Verurteilungen zu unbedingten Freiheitsstrafen erkennen, wo sich die Anzahl der Verurteilungen mit 23 gegenüber 12 in der Ausgangsstudie beinahe verdoppelt hat. Allerdings ist der Anteil mit 33\% (ohne Berücksichtigung der mittlerweile verstorbenen acht Probanden) auch nach so vielen Jahren noch immer nicht höher als bei regulären Entlassungen aus dem Strafvollzug in einem Beobachtungszeitraum von vier Jahren (erneute Freiheitsstrafe ohne Bewährung in 31,36\% aller Fälle ${ }^{3}$ ).

Von ursprünglich 50 Probanden, die bis zum 30.06.2008 nicht erneut mit Delinquenz registriert worden waren $(65 \%)$, sind unter Vernachlässigung der Todesfälle bis zum 30.06.2013 lediglich 27 übrig geblieben (40\%). Bei 17 Haftentlassenen, die bis zum 30.06.2008 nicht mit Rückfalldelinquenz registriert worden waren, ist nunmehr mindestens ein Rückfalldelikt erfasst. Weitere 17 Probanden, die bereits am 30.06.2008 mit Rückfällen erfasst waren, sind erneut bestraft worden, acht seinerzeit Registrierte sind nicht noch einmal aufgefallen, befinden sich aber teilweise auch noch in Sicherungsverwahrung. Die Entwicklung ist im Hinblick auf erneute Verurteilungen zu Freiheitsstrafen von mehr als zwei Jahren Dauer aus der folgenden Tabelle ersichtlich:

\section{Index-und Rückfalldelikt bei Verurteilung zu Freibeitsstrafe von mebr als zwei Jabren und Strafmaß}

\begin{tabular}{|c|c|c|c|c|}
\hline Indexdelikt & $\begin{array}{c}\text { Rückfalldelikt } \\
\text { bis 30.06.2008 re- } \\
\text { gistriert }\end{array}$ & Strafmaß & $\begin{array}{c}\text { Rückfalldelikt } \\
\text { bis 30.06.2013 re- } \\
\text { gistriert }\end{array}$ & Strafmaß \\
\hline Vergewaltigung & $\begin{array}{c}\text { Gemeinsch. Raub, } \\
\text { gef. Körperverletzg. }\end{array}$ & 2 Jahre 2 Monate & ----- & ---- \\
\hline $\begin{array}{c}\text { Versuchte Verge- } \\
\text { waltigung }\end{array}$ & $\begin{array}{c}\text { Gemeinschaftl. } \\
\text { Diebstahl }\end{array}$ & 2 Jahre 6 Monate & Diebstahl, Betrug & 2 Jahre 9 Monate \\
\hline Sexuelle Nötigung & $\begin{array}{c}\text { Sexuelle Nötigung, } \\
\text { Körperverletzung }\end{array}$ & $\begin{array}{c}\text { 3 Jahre und Si- } \\
\text { cherungsverw. }\end{array}$ & ----- & ---- \\
\hline $\begin{array}{c}\text { Sex. Missbrauch } \\
\text { von Kindern }\end{array}$ & ----- & ----- & $\begin{array}{c}\text { Vers. schw. sex. } \\
\text { Missbrauch }\end{array}$ & $\begin{array}{c}\text { 3 Jahre 2 Monate } \\
\text { und Sicherungsv. }\end{array}$ \\
\hline
\end{tabular}

3 Jeble et al. 2003, 72.

NK 25. Jg. 4/2013 


\begin{tabular}{|c|c|c|c|c|}
\hline Indexdelikt & $\begin{array}{c}\text { Rückfalldelikt } \\
\text { bis } \begin{array}{c}30.06 .2008 \text { re- } \\
\text { gistriert }\end{array}\end{array}$ & Strafmaß & $\begin{array}{c}\text { Rückfalldelikt } \\
\text { bis 30.06.2013 re- } \\
\text { gistriert }\end{array}$ & Strafmaß \\
\hline $\begin{array}{c}\text { Diebstahl, Fahren } \\
\text { o.FE }\end{array}$ & $\begin{array}{c}\text { Gewerbsm. Handel } \\
\text { BtM }\end{array}$ & 3 Jahre 6 Monate & ----- & ----- \\
\hline $\begin{array}{l}\text { Vergewaltigung, } \\
\text { fahrl. Tötung }\end{array}$ & ----- & ----- & $\begin{array}{l}\text { Vers. schwere } \\
\text { räub. Erpressg. }\end{array}$ & $\begin{array}{l}3 \text { Jahre } 9 \text { Monate } \\
\text { und Sicherungsv. }\end{array}$ \\
\hline $\begin{array}{l}\text { Sex. Missbrauch } \\
\text { von Kindern }\end{array}$ & ----- & ----- & $\begin{array}{l}\text { Sex. Missbrauch } \\
\text { von Kindern }\end{array}$ & $\begin{array}{l}3 \text { Jahre } 9 \text { Monate } \\
\text { und Sicherungsv. }\end{array}$ \\
\hline $\begin{array}{l}\text { Räub. Angriff auf } \\
\text { Kraftf., schw. räub. } \\
\text { Erpr.,Geiselnahme }\end{array}$ & $\begin{array}{l}\text { Betrug, Einbruchs- } \\
\text { diebst., Urkunden- } \\
\text { fälschung }\end{array}$ & 4 Jahre & $\begin{array}{l}\text { Bes. schwerer } \\
\text { Raub }\end{array}$ & 9 Jahre \\
\hline $\begin{array}{l}\text { Sex. Missbrauch } \\
\text { von Kindern }\end{array}$ & ----- & ----- & $\begin{array}{l}\text { Sex. Missbrauch } \\
\text { von Kindern }\end{array}$ & $\begin{array}{l}5 \text { Jahre und vor- } \\
\text { beh. SV }\end{array}$ \\
\hline $\begin{array}{l}\text { Schw. Brandst., } \\
\text { Körperverletzung }\end{array}$ & ----- & ----- & $\begin{array}{l}\text { Vers. schw. } \\
\text { Brandstiftg., KV }\end{array}$ & $\begin{array}{l}6 \text { Jahre und Si- } \\
\text { cherungsverw. }\end{array}$ \\
\hline $\begin{array}{c}\text { Räub. Angriff auf } \\
\text { Kraftf., vers. schw. } \\
\text { Raub }\end{array}$ & ----- & ----- & $\begin{array}{c}\text { Diebstahl, } \\
\text { Computerbetrug }\end{array}$ & 6 Jahre 9 Monate \\
\hline $\begin{array}{l}\text { Schw. sex. Miss- } \\
\text { brauch von Kin- } \\
\text { dern }\end{array}$ & $\begin{array}{l}\text { Schw. sex. Miss- } \\
\text { brauch von Kindern }\end{array}$ & $\begin{array}{l}7 \text { Jahre und } \\
\text { Sicherungsverw. }\end{array}$ & ----- & ----- \\
\hline $\begin{array}{l}\text { Gemeinsch. schw. } \\
\text { Raub }\end{array}$ & ----- & ----- & $\begin{array}{l}\text { Vergewaltigung, } \\
\text { Körperverlet- } \\
\text { zung }\end{array}$ & $\begin{array}{l}7 \text { Jahre } 3 \text { Monate } \\
\text { und Sicherungsv. }\end{array}$ \\
\hline $\begin{array}{l}\text { Schwere räub. Er- } \\
\text { pressung }\end{array}$ & $\begin{array}{c}\text { Schwere räub. Er- } \\
\text { pressung } \\
\text { BtMG }\end{array}$ & $\begin{array}{l}11 \text { Jahre und } \\
\text { Sicherungsverw. }\end{array}$ & ----- & ----- \\
\hline
\end{tabular}

Im Vergleich zur Ersterhebung im Jahre 2008 sind somit zu den ursprünglich mit erheblicher Rückfalldelinquenz (Verurteilung zu Freiheitsstrafe von mehr als zwei Jahren) aufgefallenen sieben Haftentlassenen weitere sieben Haftentlassene hinzu getreten (Zwei Probanden waren bereits 2008 registriert und sind danach erneut mit gravierender Delinquenz aufgefallen). Drei der insgesamt 14 Haftentlassenen mit erheblicher Rückfalldelinquenz sind allerdings ausschließlich wegen Vermögensdelikten verurteilt worden. Damit verbleiben elf Probanden, die wegen Katalogtaten des $\$ 66$ b verurteilt worden, in deren Taten sich also die dort geforderte Erheblichkeit für die Integrität der Opfer aufzeigen lässt (16\% unter Vernachlässigung der acht bereits verstorbenen Probanden). Bei regulärer Entlassung aus dem Strafvollzug beträgt die entsprechende Rückfallquote 26,85\% nach Verurteilung wegen Raubes/räuberischer Erpressung, 10,33\% nach Verurteilung wegen Tötungsdelikten und 19,47\% nach Verurteilung wegen Vergewaltigung/sexueller Nötigung ${ }^{4}$. Haftentlassene, die wegen Tötungsdelikten verurteilt worden

4 Jeble et al. 2003, 72. 
waren $(n=15)$ sind nach der obigen Tabelle überhaupt nicht mit schwerwiegender Rückfalldelinquenz aufgefallen. Das Ausmaß der Gewalt bei den ansonsten aufgeführten Rückfalltaten entspricht dem Vorgehen bei der Anlassverurteilung, auch wenn nicht alle Rückfälle einschlägig sind. Weitgehend identische Vorgehensweisen lassen sich bei fünf Sexualdelikten, einem Fall von Brandstiftung (fiel seinerzeit nicht unter $\$ 66 \mathrm{~b}$ ) und zwei Fällen von schwerer räuberischer Erpressung/Raub erkennen, wobei bei Vermögensund Raubdelikten auch bei den Wiederholungstaten in sieben Fällen auf die Anordnung von Sicherungsverwahrung verzichtet wurde. Angesichts der aktuellen Diskussion um die Prävention nach Verurteilung wegen Sexualstraftaten sind insbesondere die entsprechenden fünf Rückfalldelikte einer besonderen Beachtung wert. In die Untersuchung waren 45 wegen Sexualstraftaten Verurteilte einbezogen (25 wegen sexuellen Missbrauchs Verurteilte, 20 wegen Vergewaltigung/sexueller Nötigung Verurteilte), sie stellten mit 58\% die Mehrheit der in die Untersuchung einbezogenen Haftentlassenen. Fünf einschlägige Rückfälle ergeben unter diesen Umständen eine Rückfallquote von $12 \%$ (drei wegen Sexualdelikten Verurteilte waren zum zweiten Erhebungszeitpunkt bereits verstorben), so dass auch unter Berücksichtigung eines nunmehr mindestens $61 / 2$ jährigen Beobachtungszeitraums von einer besonderen Bedrohung durch Sexualstraftaten keine Rede sein kann. Bei regulärer Entlassung aus dem Strafvollzug werden innerhalb von drei Jahren $12 \%$ der wegen sexuellen Missbrauchs Verurteilten einschlägig rückfällig und 3\% der wegen sexueller Gewalt Verurteilten ${ }^{5}$. Bei der in der Übersicht erfassten Vergewaltigung im Falle eines zuvor wegen gemeinschaftlichen schweren Raubes Verurteilten handelt es sich um ein Beziehungsdelikt (Vergewaltigung der bisherigen Lebensgefährtin).

Bei sechs der mit erheblicher Rückfalldelinquenz aufgefallenen 14 Haftentlassenen hatten die Sachverständigen übereinstimmend eine hohe Gefährlichkeit bejaht, allerdings auch bei 26 Probanden, die nicht wieder gravierend aufgefallen sind.

\section{Die Nachfolgeuntersuchung von 2011}

Die für 2011 vorgesehene Wiederholung des Zentralregisterabgleichs bot darüber hinaus die ursprünglich nicht ins Auge gefasste Möglichkeit, den Umfang der Untersuchung auf potentielle Kandidaten für die nachträgliche Anordnung von Sicherungsverwahrung, die nach Ablehnung entsprechender Anträge der Staatsanwaltschaft zwischen dem 01.01.2007 und dem 31.12.2009 aus dem Strafvollzug entlassen worden waren, auszudehnen. Erneut wurde über die Landesjustizverwaltungen in den Bundesländern und die Generalstaatsanwaltschaften um Auskunft über entsprechende Fälle gebeten, nach Auskunftserteilung wurden die Vollstreckungsunterlagen der betreffenden Haftentlassenen durchgesehen. Insgesamt wurden 54 entsprechende Fälle gemeldet. Im August 2012 gab das Bundesamt für Justiz dem Antrag auf Auskunftserteilung vom 09.07.2011 bezüglich der Erstauskünfte statt und übersandte 52 Auszüge aus dem Bundeszentralregister (zwei Entlassene waren zu diesem Zeitpunkt bereits verstorben). Zu diesem Zeitpunkt hatten

5 Jeble et al. 2010, $128 \mathrm{ff}$.

NK 25. Jg. 4/2013 
sich 27 Probanden bereits vier Jahre und länger in Freiheit befunden, 16 drei bis vier Jahre und 9 zweieinhalb bis drei Jahre. Der durchschnittliche Beobachtungszeitraum lag bei 48 Monaten, so dass die Bedenken gegen die teilweise zu geringe Dauer zwischen Haftentlassung und Abgleich mit dem Bundeszentralregister bei der Ausgangsuntersuchung für die Nachfolgestudie nicht gelten, zumal sich fast alle gravierenden Rückfälle in den ersten 18 Monaten nach der Entlassung ereigneten ${ }^{6}$.

Im Hinblick auf die Rückfallhäufigkeit ergab sich diesmal folgendes Bild:

Strafhöhe bei erneuter Verurteilung $(n=21)$

\begin{tabular}{|c|c|c|c|c|c|c|c|}
\hline \multicolumn{7}{|c|}{ Erneute Verurteilung zu } & \multirow{3}{*}{$\begin{array}{c}\text { Keine erneute } \\
\text { Eintragung } \\
\text { bis } 23.08 .2012\end{array}$} \\
\hline \multirow[t]{2}{*}{ Geldstrafe } & \multirow[t]{2}{*}{$\begin{array}{l}\text { Freiheitsstrafe } \\
\text { m. Bewährung }\end{array}$} & \multicolumn{4}{|c|}{$\begin{array}{l}\text { Freiheitsstrafe o. Bewäh- } \\
\text { rung }\end{array}$} & \multirow[t]{2}{*}{$\begin{array}{c}\text { Sonstiges } \\
(\mathbb{S} 63,67 \mathrm{~h} \mathrm{StGB})\end{array}$} & \\
\hline & & $<1 \mathrm{~J}$ & $\leq 2 \mathrm{~J}$ & $>2 \mathrm{~J}$ & SV & & \\
\hline 4 & 2 & 2 & 2 & 1 & 8 & 2 & 31 \\
\hline
\end{tabular}

Einzelheiten zu den neun schwerwiegenderen Rückfalltaten (Verurteilung zu Freiheitsstrafe von mehr als zwei Jahren) ergeben sich aus der folgenden Tabelle:

\section{Index- und Rückfalldelikt bei Verurteilung zu Freibeitsstrafe von mehr als zwei Jabren und Strafmaß}

\begin{tabular}{|c|c|c|}
\hline Indexdelikt & Rückfalldelikt & Strafmaß \\
\hline $\begin{array}{l}\text { Sex. Missbrauch von Kin- } \\
\text { dern, Vergewaltigung }\end{array}$ & Schwerer Diebstahl & 4 Jahre \\
\hline Schwere räub. Erpressung & Schwere räuberische Erpressung & 3 Jahre und Sicherungsverwahrung \\
\hline $\begin{array}{c}\text { Schwerer sex. Miss- } \\
\text { brauch, Vergewaltigung }\end{array}$ & $\begin{array}{l}\text { Schwerer sex. Missbrauch von Kin- } \\
\text { dern }\end{array}$ & $\begin{array}{l}3 \text { Jahre } 6 \text { Monate und Sicherungsver- } \\
\text { wahrung }\end{array}$ \\
\hline $\begin{array}{l}\text { Schwerer sex. Miss- } \\
\text { brauch. }\end{array}$ & $\begin{array}{c}\text { Schwerer sex. Missbrauch von Kin- } \\
\text { dern }\end{array}$ & $\begin{array}{c}4 \text { Jahre } 6 \text { Monate und Sicherungsver- } \\
\text { wahrung }\end{array}$ \\
\hline $\begin{array}{l}\text { Mord, vers. Totschlag, } \\
\text { Raub, }\end{array}$ & Gefährliche Körperverletzung & $\begin{array}{c}4 \text { Jahre } 6 \text { Monate und Sicherungsver- } \\
\text { wahrung }\end{array}$ \\
\hline $\begin{array}{l}\text { Gefährliche Körperver- } \\
\text { letzung }\end{array}$ & Gefährliche Körperverletzung & $\begin{array}{l}6 \text { Jahre } 6 \text { Monate und } \\
\text { Sicherungsverwahrung }\end{array}$ \\
\hline Mord, schwerer Raub & Schwere räuberische Erpressung & $\begin{array}{l}9 \text { Jahre } 6 \text { Monate und } \\
\text { Sicherungsverwahrung }\end{array}$ \\
\hline $\begin{array}{l}\text { Sex. Missbrauch von Kin- } \\
\text { dern }\end{array}$ & $\begin{array}{l}\text { Vergewaltigung, schwerer sex. } \\
\text { Missbrauch von Kindern }\end{array}$ & $\begin{array}{l}\text { 11 Jahre } 9 \text { Monate und Sicherungsver- } \\
\text { wahrung }\end{array}$ \\
\hline $\begin{array}{l}\text { Körperverletzung, Be- } \\
\text { drohung }\end{array}$ & Versuchter Mord, Vergewaltigung & 13 Jahre und Sicherungsverwahrung \\
\hline
\end{tabular}

6 Die Gesamtergebnisse sind veröffentlicht bei Alex 2013. 
Acht Rückfalldelikte sind Katalogtaten des $\$ 66 \mathrm{~b}$ StGB und wurden bei der erneuten Verurteilung durchgängig mit der Anordnung von Sicherungsverwahrung sanktioniert. Der Anteil der Probanden, bei denen sich die angenommene hohe Gefährlichkeit in so gravierender Form manifestierte, beträgt 15\%, was den Befunden bei der im Jahre 2013 erfolgten Nachuntersuchung bezüglich der bis spätestens 31.12.2006 aus dem Strafvollzug Entlassenen entspricht. Beide Untersuchungen kommen somit zum Ergebnis, dass bei 85\% der zum letzten Erhebungszeitpunkt noch lebenden 121 Haftentlassenen mit prognostizierter hoher Gefährlichkeit die vermeintliche Gefährlichkeit sich nicht in schwerer Gewalt- oder Sexualdelinquenz niedergeschlagen hat. Im Hinblick auf Sexualdelinquenz deutet die Zusammenfassung beider Untersuchungen darüber hinaus interessante Aspekte zur Altersstruktur bei Rückfalldelinquenz an, wie aus der folgenden Übersicht hervorgeht:

Altersstruktur und Delinquenz $(n=121)$

\begin{tabular}{|c|c|c|c|c|c|c|}
\hline \multirow{2}{*}{$\begin{array}{l}\begin{array}{c}\text { Alter bei Ent- } \\
\text { lassung }\end{array} \\
\text { 40 Jahre }\end{array}$} & \multicolumn{2}{|c|}{$\begin{array}{l}\text { Sexualdelikt als } \\
\text { Indexdelikt }\end{array}$} & \multirow{2}{*}{$\begin{array}{c}\begin{array}{l}\text { Rückfalldelikt } \\
\text { mit FS o. Bew. }\end{array} \\
10\end{array}$} & \multicolumn{2}{|c|}{$\begin{array}{l}\text { Sonstiges Index- } \\
\text { delikt }\end{array}$} & \multirow{2}{*}{$\begin{array}{c}\text { Rückfalldelikt mit } \\
\text { FS o. Bew. } \\
12\end{array}$} \\
\hline & 23 & $33,8 \%$ & & 32 & $60,4 \%$ & \\
\hline$\leq 50$ Jahre & 27 & $39,7 \%$ & 5 & 14 & $26,4 \%$ & 5 \\
\hline \multirow[t]{2}{*}{$>50$ Jahre } & 18 & $26,5 \%$ & 1 & 7 & $13,2 \%$ & 4 \\
\hline & \multicolumn{2}{|c|}{68} & 16 & \multicolumn{2}{|c|}{53} & 21 \\
\hline
\end{tabular}

Auffällig ist zunächst, dass zum Zeitpunkt der Entlassung zwei Drittel der wegen Sexualstraftaten Verurteilten älter als 40 Jahre waren, aber nur knapp 40\% der Verurteilten mit einem anderen Indexdelikt. Dies ist weniger auf die Strafhöhe zurückzuführen als auf die unterschiedliche Deliktsstruktur. Wie die jährlichen Stichtagserhebungen der Kriminologischen Zentralstelle in Wiesbaden zur Sozialtherapie ${ }^{7}$ verdeutlichen, hat sich auch in den sozialtherapeutischen Einrichtungen mit der zunehmenden Konzentration auf Sexualstraftaten seit dem „Sexualstraftäterbekämpfungsgesetz“ von 1998 die Altersstruktur nach oben verschoben. Insbesondere in Fällen sexuellen Missbrauchs werden die Taten häufig erst in späteren Jahren begangen, wenn die familiären oder beruflichen Voraussetzungen geschaffen sind, und häufig von den Opfern erst spät angezeigt, wenn die Abhängigkeit vom Täter geringer geworden ist. 37 der 68 wegen Sexualstraftaten Verurteilten waren wegen sexuellen Missbrauchs verurteilt worden.

Betrachtet man jedoch die Zahl von Rückfällen, die mit unbedingter Freiheitsstrafe geahndet wurden, so unterscheiden sich die beiden Gruppen nicht. Bemerkenswert ist dabei auch, dass nur einer von $18 \mathrm{im}$ Alter von mehr als 50 Jahren entlassenen Sexualstraftätern erheblich rückfällig wurde (versuchter schwerer sexueller Missbrauch von Kindern). Die alltagstheoretische Vorstellung, dass Sexualstraftäter auch in hohem Alter

7 Abrufbar unter: www.krimz.de.

NK 25. Jg. 4/2013 
rückfallgefährdeter sind als andere Tätergruppen, findet in diesen Daten keine Bestätigung.

\section{Diskussion}

Gegenstand der Untersuchung war die Rückfallhäufigkeit von Verurteilten, denen am Ende des Strafvollzugs eine so hohe Gefährlichkeit für die Begehung schwerer Gewaltoder Sexualdelikte prognostiziert worden war, dass bei ihnen die nachträgliche Anordnung von Sicherungsverwahrung gemäß $\ 66 \mathrm{~b}$ StGB angestrebt, letztlich aber bei Gericht, teilweise auch durch Rücknahme der Anträge durch die Staatsanwaltschaft, nicht durchgesetzt werden konnte, und die deshalb trotz der vermeintlichen Gefährlichkeit aus dem Strafvollzug entlassen worden sind. Die Ergebnisse der Untersuchung zeigen, dass $15,7 \%$ von ihnen nach der Entlassung mit der von Sachverständigen erwarteten erheblichen Gewalt- oder Sexualdelinquenz aufgefallen sind, wohingegen die übrigen Haftentlassenen entweder überhaupt nicht $(47,9 \%)$ oder überwiegend nur mit geringfügiger Delinquenz, die nicht zu erneuter Inhaftierung führte, registriert worden sind $(20,7 \%) .17$ weitere Probanden (14\%) wurden wegen gewaltloser Delinquenz zu unbedingten Freiheitsstrafen verurteilt, vier von ihnen zu Strafen von mehr als zwei Jahren. Auch nach einem Beobachtungszeitraum von mehr als $61 \frac{1}{2}$ Jahren sind nur neun Haftentlassene so massiv mit Gewalt- oder Sexualdelinquenz aufgefallen, dass anlässlich der neuen Verurteilung zusätzlich (vorbehaltene) Sicherungsverwahrung angeordnet worden ist, im gleichen Zeitraum sind aber auch acht der Entlassenen verstorben.

Die Befunde machen deutlich, dass Prognosen zur künftigen Legalbewährung von Haftentlassenen auch nach Einführung von Standards für Prognosegutachten ${ }^{9}$ weiterhin sehr unsicher sind, ganz zu schweigen von der Unmöglichkeit, menschliches Verhalten für lange Zeiträume vorherzusagen. Ohne konkreten Befund wird kein Sachverständiger sich ernsthaft mit der Frage beschäftigen, ob möglicherweise durch den nahen Tod des Begutachteten eine Rückfallgefährdung ausgeschlossen ist, obwohl nach den Ergebnissen unserer Untersuchung die Wahrscheinlichkeit dafür ebenso groß ist wie die, dass es tatsächlich zu dem erwarteten schwerwiegenden Rückfall kommt. Das zugegebenermaßen weit hergeholte Beispiel macht darauf aufmerksam, dass das künftige Legalverhalten häufig von Variablen außerhalb der Persönlichkeit des Betroffenen, wie Qualität der Entlassungsvorbereitung, das Eingehen oder Scheitern von Beziehungen, Arbeitsaufnahme oder Arbeitslosigkeit abhängt, so dass sich hier langfristig protektive oder destabilisierende Faktoren auswirken, deren Bedeutung bei einer Begutachtung vor Haftentlassung überhaupt nicht abschätzbar ist. Angesichts der geringen Quote von Neueinträgen von erheblicher Bedeutung im Bundeszentralregister ist davon auszugehen, dass die Gefährlichkeit von nach vielen Jahren aus der Haft entlassenen Verurteilten durch die beigezogenen Sachverständigen weit überschätzt wird. Das kann mit der Profession der beauftragten Sachverständigen zusammenhängen, deren Blick weniger auf kriminogene

9 Boetticher et al. 2010, 537-544. 
Faktoren als auf psycho-pathologische Persönlichkeitszüge gerichtet ist, es dürfte aber vorrangig auf die trotz aller methodischen Verbesserungen weiterhin unzureichenden Möglichkeiten einer zuverlässigen Gefährlichkeitsprognose zurückzuführen sein. Kriminalprognose bleibt ein Feld, dem wissenschaftstheoretisch und methodisch enge Grenzen gesetzt sind. Statische Faktoren aus der Vergangenheit werden von Veränderungen im Alterungsprozess überlagert, eine gute Entlassungsvorbereitung kann einen sozialen Empfangsraum in einem protektiven Umfeld aufbauen, vermeintlich protektive Faktoren können nach der Entlassung durch Beziehungsabbrüche oder ähnliche Veränderungen ihre schützende Wirkung verlieren ${ }^{10}$. Mit der Erarbeitung von Standards für die Gutachtenerstellung und der Verbesserung des diagnostischen Instrumentariums hat vor allem die Psychiatrie bei den Entscheidungsträgern - bis hin zum Bundesverfassungsgericht - viel zu hohe Erwartungen an die Zuverlässigkeit der abgegebenen Prognosen geweckt. Allzu vorsichtig sind die Unsicherheiten bezüglich der Prognosestellung formuliert worden, so dass es der Politik zuletzt leicht fiel, Sicherungsverwahrung sogar für nach Jugendstrafrecht Verurteilte einzuführen, nachdem vor gerade einmal 20 Jahren die wissenschaftlichen Erkenntnisse zur Abschaffung der unbestimmten Jugendstrafe geführt hatten. Statt immer wieder darauf hinzuweisen, wie valide die neuen Instrumente der Prognosebeurteilung sind, sollten forensische Psychiatrie und Psychologie viel deutlicher herausstellen, wie hoch die Zahl der „falschen Positiven“ ist, also der Anteil der Ungefährlichen, die mit Rücksicht auf das vermeintliche Sicherheitsbedürfnis der Bevölkerung unberechtigt auf Dauer in psychiatrischen Krankenhäusern oder Justizvollzugsanstalten eingesperrt sind. Solange sich forensische Psychiatrie und forensische Psychologie nicht erkennbar dem symbolischen Spiel der Politik, „Sicherheit vor Kinderschändern" zur Kompensation für eine allgemeine Verunsicherung der Gesellschaft zu missbrauchen, verweigern, machen sie sich zum Komplizen der Ausgrenzungsstrategie.

Auch die insbesondere in der Politik vorhandene Vorstellung, dass Sexualdelikte oft erst viele Jahre nach der Entlassung aus dem Strafvollzug begangen werden und dass nach Verurteilungen wegen Sexualstraftaten das Alter die Rückfallwahrscheinlichkeit nicht zu vermindern vermag, ist nach den Ergebnissen unserer Untersuchung allenfalls für Fälle sexuellen Missbrauchs aufrecht zu erhalten. So haben sich drei der vier einschlägigen Fälle sexuellen Missbrauchs erst drei bis fünf Jahre nach der Entlassung ereignet, eines der insgesamt sechs Sexualdelikte wurden aber innerhalb eines Jahres und die übrigen zwei innerhalb von zwei Jahren begangen. Andererseits ist auch die versuchte schwere Brandstiftung erst zwei Jahre und vier Monate nach der Entlassung begangen worden, zwei der Raubdelikte im zweiten Jahr nach den Entlassung, die übrigen zwei in den ersten vier Monaten. Im Hinblick auf die Altersstruktur bleibt festzuhalten, dass ab einem Alter von mehr als 50 Jahren kaum noch schwerwiegende Straftaten begangen werden und Sexualstraftaten jedenfalls keineswegs überrepräsentiert sind, sondern von dieser Altersgruppe extrem selten begangen werden (ein Fall bei 18 Vorbestraften).

10 Vgl. zur Bedeutung protektiver Faktoren Nowara 2006, $182 \mathrm{f}$.

NK 25. Jg. 4/2013 


\section{E. Fazit}

Die Ergebnisse der Rückfalluntersuchung zur nachträglichen Sicherungsverwahrung bestätigen auch nach Ausweitung der Studie in Umfang und Beobachtungsdauer, dass es für die hysterischen Gesetzesinitiativen der vergangenen 15 Jahre keinen rationalen Hintergrund gibt. Die einschlägigen Rückfallquoten vermeintlich hoch gefährlicher Gewaltund Sexualstraftäter sind marginal, das Vertrauen in die Zuverlässigkeit von Gefährlichkeitsprognosen ist nicht gerechtfertigt. Nur bei einer äußerst geringen Zahl der jährlich wegen Sexualdelikten, Mord/Totschlags oder Raubes ca. 25.000 Abgeurteilten ${ }^{11}$ handelt es sich um Rückfalltäter, etwa $85 \%$ betreffen Täter, die mit derartigen Delikten bisher nicht aufgefallen waren.

Konsequenz daraus muss sein, die Debatte um den Umgang mit verurteilten Sexualoder Gewaltstraftätern zu versachlichen und die Gerichte von der Abhängigkeit von Gutachtern zu befreien. Vermeintlich hoch gefährliche Haftentlassene fallen nur in Ausnahmefällen erneut mit einschlägiger Gewalt- oder Sexualdelinquenz auf, aber dennoch ist der Fokus von Politik und Bevölkerung seit 1998 ausschließlich auf diesen Personenkreis gerichtet. Wird bekannt, dass entlassene Sexualstraftäter sich in der Nachbarschaft niederlassen wollen, gibt es sofort massive Proteste zur Einschüchterung (markanteste Beispiele sind Heinsberg in Nordrhein-Westfalen und Insel in Sachsen-Anhalt, aber auch Großstädte wie Hamburg, wo jegliche Resozialisierungsbemühungen torpediert werden), will ein Verurteilter zur Entlassungsvorbereitung wieder in seinem früheren Betrieb arbeiten, sträuben sich die ehemaligen Kollegen mit Unterstützung von Firmenleitung und Betriebsrat(!) durch Streikaktionen erfolgreich gegen die Wiedereinstellung (Bremerhaven, August 2013). Der Politik kommt dieser Aufruhr meist entgegen, lenkt die Fixierung auf entlassene Sexualstraftäter doch ab von vielen anderen Problemen einer gespaltenen Gesellschaft, in der die Schere zwischen Arm und Reich immer weiter auseinander klafft. Viele Gutachter fördern die seit 1998 entstandene Hysterie in der Bevölkerung, indem sie den zu Entlassenden eine hohe Rückfallwahrscheinlichkeit vorhersagen, wohl wissend, dass diese Prognose in den meisten Fällen unzutreffend sein wird, weil nur in wenigen Extremfällen eine einigermaßen zuverlässige Vorhersage getroffen werden kann. Aber mit einer negativen Prognose ist man im Falle eines Rückfalls auf der sicheren Seite! Umso dringlicher ist es, dass insbesondere die Psychiatrie sich gegen die Erwartung stellt, sie könne menschliches Verhalten zuverlässig voraussagen und damit Sicherheit gewährleisten. Das gilt nicht nur im Hinblick auf die (nachträgliche) Sicherungsverwahrung, sondern auch für den Maßregelvollzug allgemein. Der Fall Mollath ist nur die Spitze eines Eisbergs, mit dem zahllose Fälle unbefristeter Unterbringung in forensischen Kliniken oder Justizvollzugsanstalten verknüpft sind. Erst wenn wir als Gesellschaft bereit sind, all diesen Menschen durch Wiedereinführung einer Befristung der Unterbringung eine echte Chance auf Teilhabe am gesellschaftlichen Leben einzuräumen, kann die Kriminalprognose den angesichts der wissenschaftlichen Grenzen für Vorhersagen angemessenen Platz insbesondere bei Unterstützung präventiver Maßnah-

11 Strafverfolgungsstatistik, 2009, 2010, Tabelle 2.1.

TITEL 
men im Hinblick auf Entlassungsvorbereitung und Nachsorge einnehmen. Als letzte Instanz, die über die dauerhafte Ausgrenzung von Menschen die Hoheit hat, sollte sich vor allem die forensische Psychiatrie nicht missbrauchen lassen!

\section{Literatur}

Alex (2013) Nachträgliche Sicherungsverwahrung - ein rechtsstaatliches und kriminalpolitisches Debakel, 2. Aufl.

Boetticher/Kröber/Müller-Isberner/Böbm/Müller-Metz/Wolf (2010) Mindestanforderungen für Prognosegutachten, in: Neue Zeitschrift für Strafrecht 26.10., 537-544

Jeble/Heinz/Sutterer (2003) Legalbewährung nach strafrechtlichen Sanktionen - eine kommentierte Rückfallstatistik

Jeble/Albrecht/Hohmann-Fricke/Tetal (2010) Legalbewährung nach strafrechtlichen Sanktionen - Eine bundesweite Rückfalluntersuchung 2004 bis 2007

Nowara (2006) Gefährlichkeitsprognosen bei Maßregeln. Zur Güte von Prognosegutachten und zur Frage der Legalbewährung, in: Barton (Hrsg.): „... weil er für die Allgemeinheit gefährlich ist", $175-185$

Rasch (1999) Forensische Psychiatrie, 2. Aufl.

Kontakt:

Michael Alex

Freier Mitarbeiter

Rubr-Universität Bochum

Lebrstubl für Kriminologie

Universitätsstr. 150

44801 Bochum

michel.alex@rub.de 